\title{
Pilot demonstration of correlation-domain distributed temperature sensing using forward Brillouin scattering
}

Neisei Hayashi ${ }^{1,2,3}$, Yosuke Mizuno ${ }^{2,4^{*}}$, Kentaro Nakamura ${ }^{2}$, Chao Zhang ${ }^{3}$, Lei Jin ${ }^{3}$, Sze Yun $\mathrm{Set}^{3}$, and Shinji Yamashita ${ }^{3}$

1 The Graduate School for the Creation of New Photonics Industries, Shizuoka 431-1202, Japan

2 Institute of Innovative Research, Tokyo Institute of Technology, Yokohama 226-8503, Japan

3 Research Center for Advanced Science and Technology, The University of Tokyo, Tokyo 153-8904, Japan

4 Faculty of Engineering, Yokohama National University, Yokohama 240-8501, Japan

*E-mail: mizuno-yosuke-rg@ynu.ac.jp

Abstract: Forward Brillouin scattering (FBS) has been regarded as a key tool for distributed sensing of strain, temperature, acoustic impedance, etc. To date, FBS-based distributed sensing has been experimentally reported only by time-domain techniques. In this paper, we demonstrate correlation-domain distributed temperature sensing using cascaded FBS seeded by backward stimulated Brillouin scattering (SBS). By localizing the backward SBS using synthesized optical coherence function, cascaded FBS can also be localized. In the proof-of-concept experiment, we detect a 104-m-long heated section in a 397-m-long highly nonlinear fiber.

Backward Brillouin scattering, including spontaneous Brillouin scattering and stimulated Brillouin scattering (SBS), has been extensively exploited to develop distributed strain and temperature sensors. ${ }^{1)}$ A lot of configurations have been reported thus far, such as time-domain, ${ }^{2-6)}$ frequency-domain, ${ }^{7,8)}$ and correlation-domain techniques. ${ }^{9-15)}$ However, it is difficult for these sensors to measure the ambient parameters that do not modify the fiber core. For example, the Brillouin frequency shift (BFS) of backward Brillouin scattering is not influenced by the change in ambient acoustic impedance.

To achieve distributed acoustic impedance sensing, forward Brillouin scattering (FBS) has recently attracted significant attention. ${ }^{16-34)} \mathrm{FBS}$, which is also one of the nonlinear optical phenomena in optical fibers, is caused by the interaction between the incident light and the acoustic waves propagating not in the longitudinal direction but in the cross-sectional area of the fiber. Unlike spontaneous Brillouin scattering and SBS, 
FBS-scattered light propagates in the same direction as the incident light (therefore, it is called "forward"). FBS has been observed not only in silica single-mode fibers (SMFs) but also in some specialty optical fibers such as polarization-maintaining fibers (PMFs), ${ }^{18)}$ few-mode fibers, ${ }^{19)}$ multi-core fibers, ${ }^{20)}$ and highly nonlinear fibers (HNLFs). ${ }^{21)}$

It has been a big challenge to measure FBS in a distributed manner because of its forward-propagating nature. Stated differently, conventional distributed Brillouin sensing techniques are based on the backward-propagating nature of the scattered light, and it has been not easy to use such techniques to FBS sensing directly. In 2018, two groups demonstrated distributed FBS sensing using time-domain techniques. ${ }^{31,32)}$ They employed a co-propagated pump-probe technique, where optical pump pulse and continuous probe were used. Although the spatial resolutions achieved in their first demonstrations were approximately $100 \mathrm{~m}$, considerable efforts have been given to enhance the resolution. ${ }^{33,34)}$ However, these techniques inherently suffer from the drawbacks of time-domain techniques, such as a relatively low sampling rate and non-feasibility of random access to sensing points. Recently, we have succeeded in backward observation of FBS by seeding it with backward SBS. ${ }^{30)}$ This indicates that conventional distributed Brillouin sensing techniques might be directly employed to perform distributed FBS sensing.

In this work, we present a proof-of-concept demonstration of distributed FBS sensing based on correlation-domain technique (more specifically, Brillouin optical correlation-domain analysis (BOCDA)). We experimentally show that a 104-m-long heated section in a 397-m-long highly nonlinear fiber is detected. Although the spatial resolution and signal-to-noise ratio are not sufficient at present, our configuration will potentially allow higher-speed operation and random accessibility to sensing points. Note that this work complements our preliminary results presented in a conference. ${ }^{35)}$ The additional value of this extended paper lies in supplementing detailed information relevant to this work (motivation, experimental conditions, etc) and providing further analysis of the experimental results.

Unlike backward Brillouin scattering caused by the longitudinal acoustic modes, forward Brillouin scattering, also known as guided-acoustic-wave Brillouin scattering (GAWBS), is caused by the radial acoustic modes. The basic principle has been well documented in other literatures. ${ }^{16,27,30)}$ Stimulated FBS can be induced by a pump-probe technique, where incident pump light (frequency: $v_{0}$ ) and probe light (frequency: $v_{0}-v_{\mathrm{FBS}}$; where $V_{F B G}$ is the amount of the acoustic resonance frequency of FBS) is co-propagated in an optical fiber. Then the energy of the pump can be transferred to the probe. Note that this 
process occurs in a cascading manner; i.e., the pump power of stimulated FBS is further transferred to a higher-order acoustic resonance mode. The resultant FBS-scattered optical spectrum includes tens of peaks in the frequency range of $<1 \mathrm{GHz}$.

We have developed a new method for observing the cascaded FBS backwardly by seeding it with backward SBS. ${ }^{30)}$ When we inject pump light (frequency: $v_{0}$ ) into one end of a fiber under test (FUT) and probe light (frequency: $v_{0}-B F S+v_{F B S}$ ) into the other end, the energy of the probe light is, via SBS, transferred to the FBS seed, and consequently, cascaded FBS can be induced backwardly. Refer to Ref. 30 for the detailed principle. We have already confirmed that the spectral peaks of the cascaded FBG induced in this method show clear temperature dependence. Then, on the basis of BOCDA technique, ${ }^{9,10)}$ by frequency-modulating the pump and the probe, a correlation peak (sensing position) can be generated in the FUT, and by sweeping its location, distributed sensing of FBS (corresponding to temperature in this work) can be performed.

In the experiment, an ultraviolet curable coated 397-m-long silica HNLF (HNDS1614C A-4-3-3, Sumitomo) with a core diameter of $3.5 \mu \mathrm{m}$, a cladding diameter of $117.0 \mu \mathrm{m}$, a core refractive index of $\sim 1.46$, and a propagation loss of $\sim 0.76 \mathrm{~dB} / \mathrm{km}$ (at $1.55 \mu \mathrm{m}$ ) was used as an FUT. Its BFS at room temperature was $9.092 \mathrm{GHz}$ at $1.55 \mu \mathrm{m}$, and the Brillouin threshold power with a pump-probe configuration was approximately $13 \mathrm{dBm}$.

The experimental setup for FBS-based distributed correlation-domain temperature sensing is depicted in Fig. 1. The basic implementation is analogous to that of standard BOCDA, $\left.{ }^{9}, 10\right)$ but our setup did not involve a lock-in amplifier or a microwave frequency sweeper and simply measured the FBS spectra using an electrical spectrum analyzer (ESA). All the optical paths except the FUT were standard silica SMFs. The light source was a distributed-feedback laser diode at $1.55 \mu \mathrm{m}$ with a $1.2 \mathrm{-kHz}$ linewidth, and its output frequency was sinusoidally modulated by direct modulation of the driving current with a modulation frequency $f_{\mathrm{m}}$ and a modulation amplitude $\Delta f$. The laser output was divided into two, pump and probe, using a 3-dB optical coupler. The pump light was polarization-scrambled, amplified to $17.0 \mathrm{dBm}$ using an erbium-doped fiber amplifier (EDFA), and injected into one end of the FUT through an optical circulator. The probe light was frequency-downshifted by $9.072 \mathrm{GHz}$ (the difference of $20 \mathrm{MHz}$ from the BFS corresponds to the first-order acoustic resonance frequency), amplified to $0.0 \mathrm{dBm}$ using another EDFA, and was injected into the other end of the FUT. The optical beat signal of the pump and probe lights was, after filtered using an optical band-pass filter (BVF-300CL, Alnair; bandwidth: $10 \mathrm{GHz}$ ) to suppress the amplified spontaneous emission, converted 
into an electrical signal using a photodetector (PD; 11982A, Keysight), and then observed using an ESA with a $100-\mathrm{kHz}$ frequency resolution. The room temperature was $25^{\circ} \mathrm{C}$.

To demonstrate distributed temperature sensing, a 104-m-long section in the FUT was heated to $60^{\circ} \mathrm{C}$, as shown in Fig. 2. The modulation frequency $f_{\mathrm{m}}$ was swept from 230.0 $\mathrm{kHz}$ to $270.0 \mathrm{kHz}$, yielding a measurement range of $447 \mathrm{~m}$ according to Eq. (2) in Ref. 10. The modulation amplitude $\Delta f$ was $100 \mathrm{MHz}$, resulting in a spatial resolution of approximately $42.5 \mathrm{~m}$. The second-order correlation peak was utilized. We measured the FBS spectrum at $\sim 942 \mathrm{MHz}$, which corresponds to the 20th-order acoustic resonance mode (note that lower-order peaks exhibit higher peak powers but less significant temperature dependencies). Averaging was performed 8000 times, and the sampling rate was $3.3 \mathrm{~Hz}$.

The measured distribution of the FBS spectra is shown in Fig. 3(a). The vertical axis was normalized so that the peak power became 1 . The relative position of $0 \mathrm{~m}$ corresponds to the probe-side end of the FUT (point "A" in Figs. 1 and 2). Although the signal-to-noise ratio was not high, the FBS spectrum clearly shifted to higher frequency around the heated section. The top view only around the peak is shown in Fig. 3(b). The shift of the frequency was approximately $3.5 \mathrm{MHz}$, which moderately agreed with the temperature sensitivity of this resonance peak. ${ }^{21)}$ Note that the frequency-shifted section was $\sim 50 \mathrm{~m}$ shifted to the pump-side end of the FUT, which is probably because $\sim 50$-m-long propagation was required for the SBS-backscattered light to seed the cascaded FBS. ${ }^{30)}$ This calls for compensation of the positional shift; on this point, further study is required.

In conclusion, correlation-domain distributed temperature sensing was demonstrated using cascaded FBS seeded by backward SBS. BOCDA-based spatially resolved backward SBS enabled distributed measurement of cascaded FBS spectra. In the proof-of-concept experiment, a 104-m-long heated section in a 397-m-long HNLF was detected. It is true that, at present, the spatial resolution and the signal-to-noise ratio are not sufficient, and that the positional calibration is required. But we believe that this work is a significant technological step toward a new class of distributed FBS sensing, which will enable higher-speed operation and random access to sensing points.

\section{Acknowledgments}

This work was partly supported by JSPS KAKENHI (17H04930, 16H00902, 17J07226, and 17K14692) and by research grants from the Noguchi Institute and the Telecommunications Advancement Foundation. 


\section{References}

[1] G. P. Agrawal, Nonlinear Fiber Optics (Academic Press, San Diego, CA, 1995).

[2] T. Horiguchi and M. Tateda, J. Lightwave Technol. 7, 1170 (1989).

[3] D. Zhou, Y. Dong, B. Wang, C. Pang, D. Ba, H. Zhang, Z. Lu, H. Li, and X. Bao, Light: Sci. Appl. 7, 32 (2018).

[4] M. A. Soto, J. A. Ramírez, and L. Thévenaz, Nat. Commun. 7, 10870 (2016).

[5] T. Kurashima, T. Horiguchi, H. Izumita, S. Furukawa, and Y. Koyamada, IEICE Trans. Commun. E76-B, 382 (1993).

[6] A. Masoudi, M. Belal, and T. P. Newson, Opt. Lett. 38, 3312 (2013).

[7] D. Garus, K. Krebber, F. Schliep, and T. Gogolla, Opt. Lett. 21, 1402 (1996).

[8] A. Minardo, R. Bernini, R. Ruiz-Lombera, J. Mirapeix, J. M. Lopez-Higuera, and L. Zeni, Opt. Express 24, 29994 (2016).

[9] K. Hotate and T. Hasegawa, IEICE Trans. Electron. E83-C, 405 (2000).

[10] K. Hotate, Appl. Sci. 9, 186 (2019).

[11] K. Y. Song, M. Kishi, Z. He, and K. Hotate, Opt. Lett. 36, 2062 (2011).

[12] Y. Okawa, R. K. Yamashita, M. Kishi, and K. Hotate, Opt. Express 28, 6981 (2020).

[13] W. Zou, C. Jin, and J. Chen, Appl. Phys. Express 5, 082503 (2012).

[14] Y. Mizuno, W. Zou, Z. He, and K. Hotate, Opt. Express 16, 12148 (2008).

[15] Y. Mizuno, W. Zou, Z. He, and K. Hotate, J. Lightwave Technol. 28, 3300 (2010).

[16] R. M. Shelby, M. D. Levenson, and P. W. Bayer, Phys. Rev. B 31, 5244 (1985).

[17] A. J. Poustie, J. Opt. Soc. Am. B 10, 691 (1993).

[18] N. Nishizawa, S. Kume, M. Mori, T. Goto, and A. Miyauchi, Opt. Rev. 3, 29 (1996).

[19] T. Matsui, K. Nakajima, and F. Yamamoto, Appl. Opt. 54, 6093 (2015).

[20] H. H. Diamandi, Y. London, and A. Zadok, Optica 4, 289 (2017).

[21] N. Hayashi, K. Suzuki, S. Y. Set, and S. Yamashita, Appl. Phys. Express 10, 092501 (2017).

[22] Y. London, H. H. Diamandi, and A. Zadok, APL Photonics 2, 041303 (2017).

[23] A. Tehranchi, V. L. Iezzi, S. Loranger, and R. Kashyap, IEEE J. Quantum Electron. 52, 1 (2016).

[24] Y. Tanaka and K. Ogusu, IEEE Photonics Technol. Lett. 11, 865 (1999).

[25] Y. Tanaka, H. Yoshida, and T. Kurokawa, Meas. Sci. Technol. 15, 1458 (2004).

[26] Y. Antman, A. Clain, Y. London, and A. Zadok, Optica 3, 510 (2016).

[27] N. Hayashi, Y. Mizuno, K. Nakamura, S. Y. Set, and S. Yamashita, Opt. Express 25, 2239 (2017).

[28] P. St. J. Russell, R. Culverhouse, and F. Farahi, Electron. Lett. 26, 1195 (1990).

[29] M. S. Kang, A. Brenn, and P. St. J. Russell, Phys. Rev. Lett. 105, 153901 (2010).

[30] N. Hayashi, Y. Mizuno, H. Lee, K. Nakamura, S. Y. Set, and S. Yamashita, IEICE Electron. Express, to appear.

[31] G. Bashan, H. H. Diamandi, Y. London, E. Preter, and A. Zadok, Nat. Commun. 9, 2991 (2018).

[32] D. M. Chow, Z. Yang, M. A. Soto, and L. Thévenaz, Nat. Commun. 9, 2990 (2018). 
[33] C. Pang, Z. Hua, D. Zhou, H. Zhang, L. Chen, X. Bao, and Y. Dong, Optica 7, 176 (2020).

[34] G. Bashan, Y. London, H. H. Diamandi, and A. Zadok, Optica 7, 85 (2020).

[35] N. Hayashi, Y. Mizuno, K. Nakamura, C. Zhang, L. Jin, S. Y. Set, and S. Yamashita, 2019 Conference on Lasers and Electro-Optics (CLEO), paper AF1K.5. 


\section{Figure Captions}

Figure 1 Experimental setup of correlation-domain FBS-based distributed sensing. EDFA: erbium-doped fiber amplifier, ESA: electrical spectrum analyzer, FG: function generator, HNLF: highly nonlinear fiber, OF: optical filter, PD: photodetector, PSCR: polarization scrambler, SSBM: single-sideband modulator.

Figure 2 Structure of the FUT.

Figure 3 (a) Measured distributions of the normalized FBS spectra along the FUT. (b) Its top view around the peak. 


\section{Figures}

Figure 1

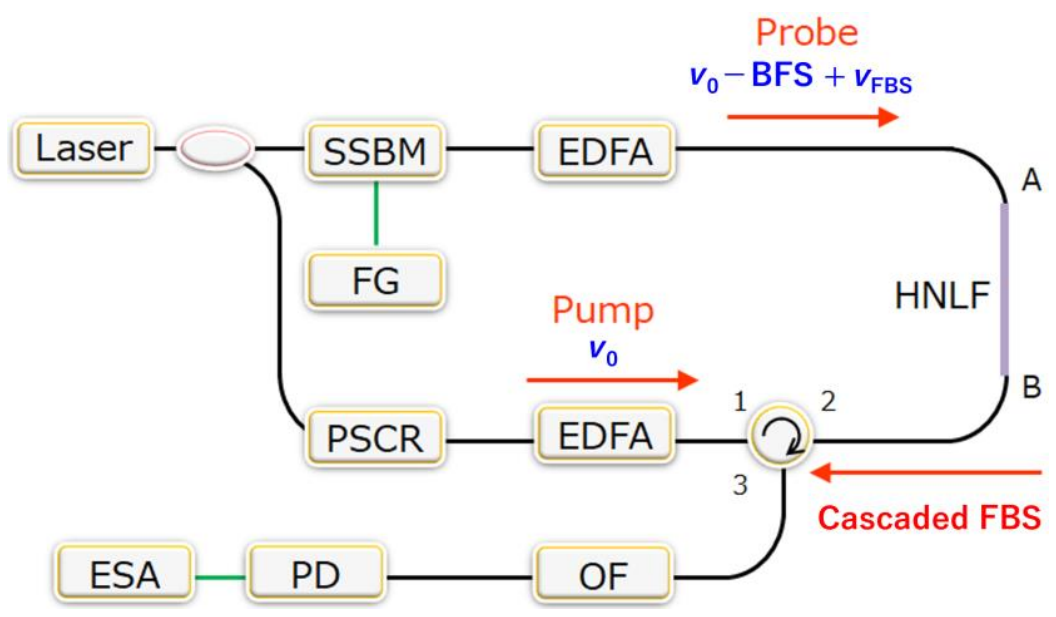

Figure 2

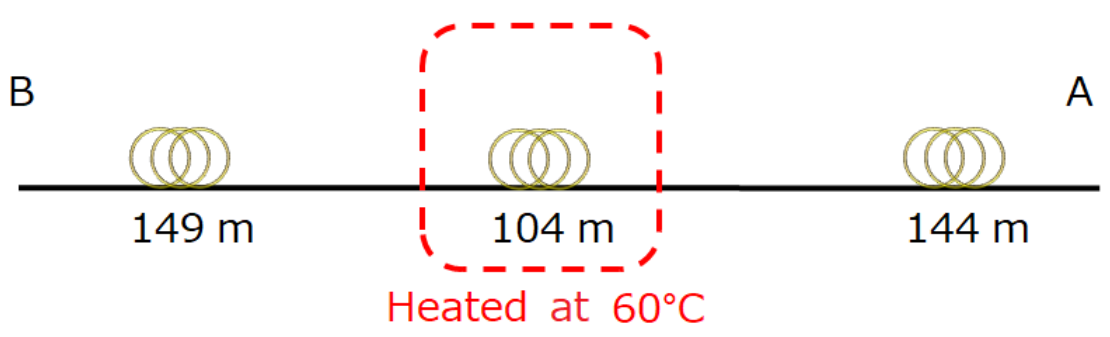

Figure 3

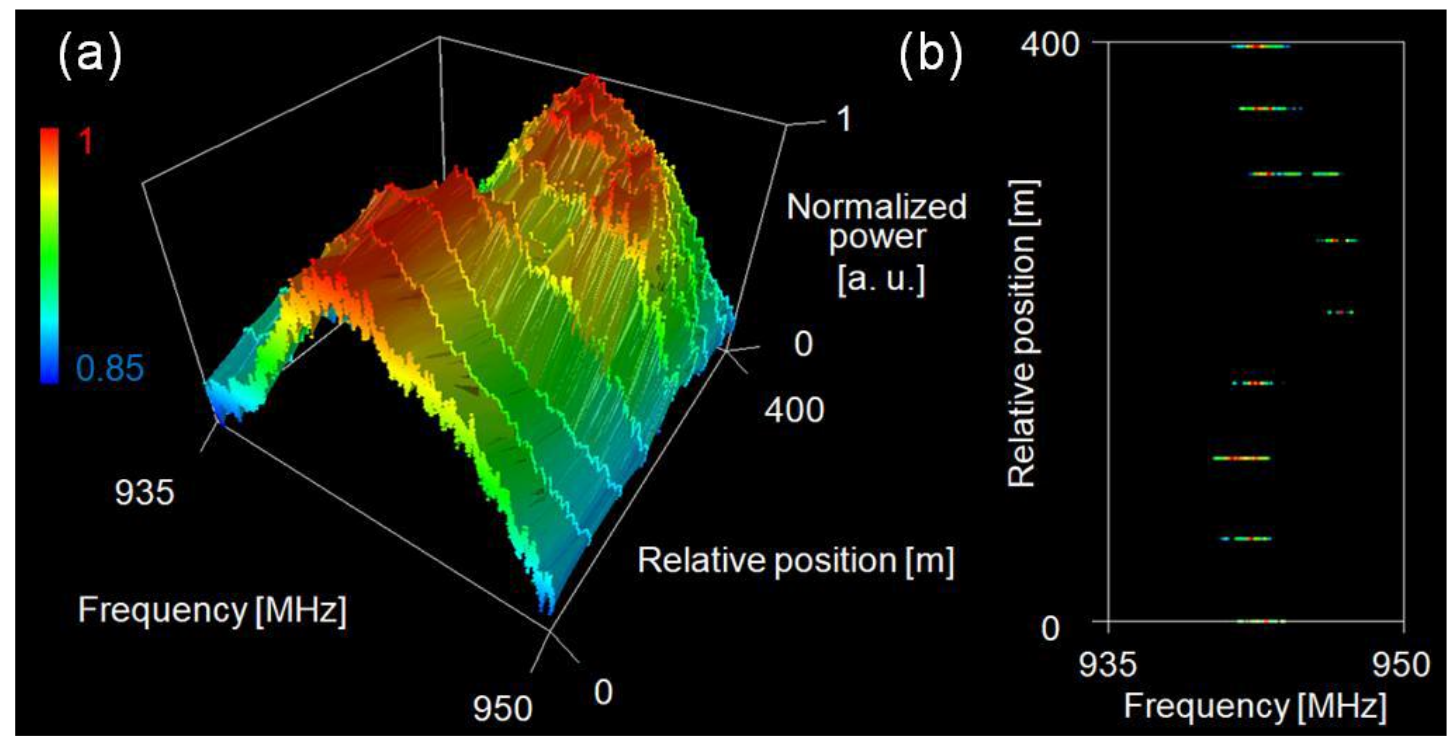

